\title{
Outbreak of Staphylococcus hominis subsp. novobiosepticus bloodstream infections in São Paulo city, Brazil
}

Coagulase-negative staphylococci (CoNS) are currently recognized as one of the most important causes of nosocomial infections worldwide, principally related to bloodstream infections (Pfaller et al., 1999). Staphylococcus epidermidis is the most frequent CoNS species associated with these infections (Rupp \& Archer, 1994), but results of surveillance studies indicate a varying frequency, depending on the geographical region (Yamazumi et al., 2001; Sader et al., 2001). Additionally, a substantial increase in the frequency of meticillin resistance among CoNS isolates has occurred over recent decades.

According to the results of the SENTRY study, about $80 \%$ of CoNS strains isolated from bloodstream infections in Brazilian hospitals are resistant to meticillin (Sader et al., 2001). Glycopeptides are usually the treatment of choice for infections caused by these micro-organisms. However, due to the emergence of vancomycin-resistant enterococci and staphylococci, reduction in the use of this antimicrobial agent has been recommended (Hiramatsu, 1998). The accurate detection of meticillinresistant CoNS isolates by clinical microbiology laboratories is of crucial importance in guiding therapy and promoting the correct use of glycopeptides (Yamazumi et al., 2001; Hussain et al., 1998). Recently, a novel subspecies of CoNS, Staphylococcus hominis subsp. novobiosepticus, was isolated from blood and other clinical specimens (Kloos et al., 1998). The subspecies name derives from the combination of novobio, pertaining to the property of novobiocin resistance, and septicus, pertaining to the ability to cause sepsis. This pathogen usually exhibits multidrug resistance, including resistance to oxacillin and other antimicrobial agents (Kloos et al., 1998; Chaves et al., 2005). Meticillin-resistant isolates and those resistant to other antimicrobials are particularly important because they narrow therapeutic options. Recently, Chaves et al. (2005) related a nosocomial outbreak caused by an S. hominis subsp. novobiosepticus clone in a neonatal intensive care unit in the city of Madrid, Spain.

From March to April 2006, three patients in the intensive care unit of Hospital Nove de Julho, a tertiary hospital located in the city of São Paulo, Brazil, presented with bloodstream infections caused by an uncommon Gram-positive coccus resistant to novobiocin. The isolates were characterized as recommended by Bannerman (2003), with some modifications. The isolates were also identified by the AutoScan (Dadebehring) and Vitek (bioMérieux) systems. The antimicrobial susceptibility patterns were determined by agar dilution according to the Clinical Laboratory Standards Institute recommendations (CLSI, 2006). The isolates were also screened for reduced susceptibility to vancomycin by testing them on brain heart infusion plates containing $6 \mu \mathrm{g}$ vancomycin $\mathrm{ml}^{-1}$ (CLSI, 2006). The genetic relatedness of isolates was evaluated by PFGE (Pfaller et al., 1992). The PFGE patterns were established by visual inspection of ethidium bromidestained gels according to Tenover criteria (Tenover et al., 1995). The presence of the gene mecA was detected by PCR. All isolates were identified as S. hominis subsp. novobiosepticus. The S. hominis subsp. novobiosepticus isolates showed resistance to oxacillin ( $\mathrm{MIC} \geqslant 256 \mu \mathrm{g} \mathrm{ml}^{-1}$ ) and teicoplanin (MIC 48-64 $\mu \mathrm{g} \mathrm{ml}^{-1}$ ) and reduced susceptibility to vancomycin (MIC 4-8 $\mu \mathrm{g} \mathrm{ml}^{-1}$ ). A unique PFGE pattern was found in the three isolates that carried the gene mecA. The predominance of a single PFGE pattern confirmed the clonal dissemination of $S$. hominis subsp. novobiosepticus.

All three patients were older than 65 years of age, presented neurological disease with more than 2 months of hospitalization, and had received teicoplanin before the isolation of S. hominis subsp. novobiosepticus. After isolation of these micro-organisms, the patients started receiving linezolid therapy. Two of them died and one was discharged from hospital after 2 months.

Our results suggest the intra-hospital dissemination of one predominant clonal group of multi-resistant $S$. hominis subsp. novobiosepticus, an uncommon pathogen.

\section{Pedro A. d'Azevedo, ${ }^{1,2}$ Regina Trancesi, ${ }^{3}$ Tiago Sales, ${ }^{4}$ Jussimara Monteiro, ${ }^{1}$ Ana C. Gales ${ }^{1}$ and Antonio C. Pignatari ${ }^{1,3}$}

\section{${ }^{1}$ Laboratório Especial de Microbiologia Clínica (LEMC) da Universidade Federal de São Paulo (UNIFESP), SP, Brazil \\ ${ }^{2}$ Laboratório de Cocos Gram Positivos da Fundação Faculdade Federal de Ciências Médicas de Porto Alegre (FFFCMPA), RS, Brazil \\ ${ }^{3}$ Comissão de Controle de Infecção do Hospital Nove de Julho, São Paulo, SP, Brazil ${ }^{4}$ Laboratório Bioclínico, São Paulo, SP,
Brazil}

Correspondence: Pedro A. d'Azevedo (pedro_dazevedo@yahoo.com.br)

Bannerman, T. L. (2003). Staphylococcus, Micrococcus, and other catalase-positive cocci that grow aerobically. In Manual of Clinical Microbiology, pp. 384-404. Edited by P. R. Murray, E. J. Baron, J. H. Jorgensen, M. A. Pfaller \& R. H. Yolken. Washington, DC: American Society for Microbiology.

Chaves, F., Garcia-Álvarez, M., Sanz, F., Alba, C. \& Otero, J. R. (2005). Nosocomial spread of a Staphylococcus hominis subsp. novobiosepticus strain causing sepsis in a neonatal intensive care unit. J Clin Microbiol 43, 4877-4879.

CLSI (2006). Performance Standards for Antimicrobial Susceptibility Testing, 16th information supplement, M100-S16. Wayne, PA: Clinical and Laboratory Standards Institute.

Hiramatsu, K. (1998). Vancomycin resistance in staphylococci. Drug Resist Updat 1, 135-150. 
Hussain, Z., Stoakes, L., Lannigan, R., Longo, S. \& Nancekivell, B. (1998). Evaluation of screening and commercial methods for detection of methicillin resistance in coagulase-negative staphylococci. J Clin Microbiol 36, 273-274.

Kloos, W. E., George, C. G., Olgiate, J. S., van Pelt, L., McKinnon, M. L., Zimmer, B. L., Müller, E., Weinstein, M. P. \& Mirrett, S. (1998). Staphylococcus hominis subsp. novobiosepticus subsp. nov., a novel trehalose-and $\mathrm{N}$-acetyl-D-glucosamine-negative, novobiocin- and multiple-antibiotic-resistant subspecies isolated from human blood cultures. Int J Syst Bacteriol 48, 799-812.

Pfaller, M. A., Hollis, R. J. \& Sader, H. S. (1992). Molecular biology - PFGE analysis of chromosomal restriction fragments. In Clinical Microbiology Procedures Handbook,

pp.10.5.c.1-10.5.c.11. Edited by H. D. Isenberg.
Washington, DC: American Society for Microbiology.

Pfaller, M. A., Jones, R. N., Doern, G. V., Sader, H. S., Kugler, K. C., Beach, M. L. \& The SENTRY Participants Group (1999). Survey of bloodstream infections attributable to gram-positive cocci: frequency of occurrence and antimicrobial susceptibility of isolates collected in 1997 in the United States, Canada, and Latin America from the SENTRY Antimicrobial Surveillance Program. Diagn Microbiol Infect Dis 33, 283-297.

Rupp, M. E. \& Archer, G. L. (1994).

Coagulase-negative staphylococci: pathogens associated with medical progress. Clin Infect Dis 19, 231-245.

Sader, H. S., Gales, A. C., Pfaller, M. A., Mendes, R. E., Zoccoli, C., Barth, A. \& Jones,
R. N. (2001). Pathogen frequency and resistance patterns in Brazilian hospitals: summary of results from three years of the SENTRY Antimicrobial Surveillance Program. Braz J Infect Dis 5, 200-214.

Tenover, F. C., Arbeit, R. D. \& Goering, R. V. (1995). Interpreting chromosomal DNA restriction patterns produced by pulsed-field gel electrophoresis: criteria for bacterial strain typing. J Clin Microbiol 33, 2233-2239.

Yamazumi, T., Furuta, I., Diekema, D. J., Pfaller, M. A. \& Jones, R. N. (2001). Comparison of the Vitek gram-positive susceptibility 106 card, the MRSA-Screen latex agglutination test, and $m e c A$ analysis for detecting oxacillin resistance in a geographically diverse collection of clinical isolates of coagulase-negative staphylococci. J Clin Microbiol 39, 3633-3636. 